\title{
Channel Selection Policy in Multi-SU and Multi-PU Cognitive Radio Networks with Energy Harvesting for Internet of Everything
}

\author{
Feng Hu, ${ }^{1,2}$ Bing Chen, ${ }^{1,2}$ Xiangping Zhai, ${ }^{1,2}$ and Chunsheng $\mathrm{Zhu}^{3}$ \\ ${ }^{1}$ The College of Computer Science and Technology, Nanjing University of Aeronautics and Astronautics, Nanjing 211106, China \\ ${ }^{2}$ The Collaborative Innovation Center of Novel Software Technology and Industrialization, Nanjing 210023, China \\ ${ }^{3}$ The Department of Electrical and Computer Engineering, The University of British Columbia, Vancouver, BC, Canada V6T $1 Z 4$ \\ Correspondence should be addressed to Bing Chen; cb_china@nuaa.edu.cn
}

Received 22 September 2016; Accepted 17 November 2016

Academic Editor: Beniamino Di Martino

Copyright (C) 2016 Feng Hu et al. This is an open access article distributed under the Creative Commons Attribution License, which permits unrestricted use, distribution, and reproduction in any medium, provided the original work is properly cited.

\begin{abstract}
Cognitive radio, which will become a fundamental part of the Internet of Everything (IoE), has been identified as a promising solution for the spectrum scarcity. In a multi-SU and multi-PU cognitive radio network, selecting channels is a fundamental problem due to the channel competition among secondary users (SUs) and packet collision between SUs and primary users (PUs). In this paper, we adopt cooperative sensing method to avoid the packet collision between SUs and PUs and focus on how to collect the spectrum sensing data of SUs for cooperative sensing. In order to reduce the channel competition among SUs, we first consider the hybrid transmission model for single SU where a SU can opportunistically access both idle channels operating either the Overlay or the Underlay model and the busy channels by using the energy harvesting technology. Then we propose a competitive set based channel selection policy for multi-SU where all SUs competing for data transmission or energy harvesting in the same channel will form a competitive set. Extensive simulations show that the proposed cooperative sensing method and the channel selection policy outperform previous solutions in terms of false alarm, average throughput, average waiting time, and energy harvesting efficiency of SUs.
\end{abstract}

\section{Introduction}

Due to the continuous development of wireless devices and services, our environment is transforming into an Internet of Everything (IoE) [1-5]. In this IoE paradigm, where everything and everyone will be connected, the bandwidth demand for limited spectrum has been greatly increasing. The scarcity of the spectrum resources has become a serious problem. This is mainly due to the traditional static spectrum allocation policy, where a particular portion of the spectrum can be only used by licensed wireless communications systems. The impoverishment of available spectrum and the underutilization of licensed spectrum facilitate the appearance of cognitive radio (CR) technology, which has evoked much enthusiasm of many scholars, and Federal Communications Commission (FCC) approved unlicensed use of licensed spectrum through CR technology [6-9].
CR has been regarded as an efficient approach to cope up with the spectrum shortage and low utilization problems [10-12]. Therefore, the introduction of cognitive radio in IoE environment can provide on-demand spectrum access among multiple devices.

Dynamic Spectrum Access (DSA) mechanism has been offered for spectrum usage. There are two major transmission models for a secondary user (SU) efficiently using idle spectrums, which are Overlay [13] and Underlay [14], respectively. In the Overlay model, a SU can exclusively and opportunistically use the licensed spectrum only if a primary user $(\mathrm{PU})$ is inactive. In other words, the SU is not allowed to access the spectrum simultaneously with the PU in order to prevent colliding with PU transmission. In contrast, even if when the PU accesses its spectrum, a SU may coexist with it as long as the interference caused to the PU by this SU does not degrade its communication quality in the 
Underlay model. However, when the PU state changes to be inactive, the transmission power of the SU will be still below the interference threshold constraint in the Underlay model. Therefore, the idle spectrum resources are not fully utilized, and the SU does not achieve optimal performance. On the other hand, when the licensed channels are very busy, the time that SU must wait for an available channel is too long, and then it may also significantly reduce the performance of Overlay model [15]. Therefore, we need to find a hybrid transmission model where the advantages of both Overlay and Underlay models are combined for the PU state variability, so that the performance of SUs can be maximized.

The hybrid transmission model has recently been proposed in [16-18]. In [16], the SU can exchange control information in the Underlay model and transmit data information in the Overlay model. However, the decision of accessing a model is not based on the sensing results. In $[17,18]$, the SU can constantly sense the activity of PUs and transmit data information in the Overlay model when the PU transmission is not detected. Otherwise, the SU reduces its transmission power to access the spectrum in the Underlay model. However, these papers did not take into account the sensing errors and neglected the effect of PU retransmission on the SU QoS. Although these related works indicated that the SU can obtain more spectrum access opportunities in the hybrid transmission model compared with the two conventional transmission models, the issue of two or more SUs competing for the same channel has rarely been studied so far to the best of our knowledge. Furthermore, the packet collision probability between SUs and a PU will increase in the multiple SUs scenario [19]. Thus, due to the importance of the collision avoidance in a CR network with multiple SUs and multiple PU channels, we need to propose a channel selection policy in the hybrid transmission model to address the collision issue.

Energy supply is always a critical issue in wireless communications. In a multi-SU and multi-PU CR network where multiple SUs access multiple PU channels in the hybrid transmission model, the SU needs to spend more energy to constantly detect many channels and switch among multiple channels. Therefore, energy efficiency is another important criterion in the CR network along with spectrum efficiency $[20,21]$. Furthermore, the cost of replacing the battery is often expensive. Recently, some energy harvesting techniques have been introduced in [22-24]. Such techniques allow devices to harvest natural sources' energy such as sun, wind, acoustic, and ambient radio frequency (RF) waves. Converting electromagnetic waves from ambient RF waves into energy is considered to be more suitable and stable for the low energy devices in sensor networks or CR networks compared with other sources [24]. Assuming that a SU is equipped with the RF energy harvesting capability, it must not only select an idle channel to transmit data but also a busy channel to harvest RF energy to obtain enough energy and spectrum usage opportunity. Hence, a suitable channel selection policy is very important to improve both the spectrum efficiency and the energy efficiency in CR networks.
Inspired by the inherent benefits of the above schemes, in this paper, we focus on the channel competition among SUs and packet collision between SUs and PUs in multiSU and multi-PU CR networks. Apart from the existing works, such as adopting the conventional noncooperative spectrum sensing method in the multi-SU CR network [25], and allowing SUs to access idle channels in the Overlay or Underlay model [14], there is no effective solution to the packet competition among multiple SUs and PUs [26]. We adopt the cooperative sensing method and the concept of competitive set to solve these two problems so that the spectrum sensing accuracy and the throughput of multiple SUs can be improved. It is noted that, in our study, SUs can harvest RF energy from busy channels by using the energy harvesting technology so as to extend their battery life.

The main contributions of this paper are as follows:

(i) We use the cooperative sensing method to avoid the packet collision between SUs and PUs. In channel sensing phase, the SUs that detect the same channel exchange the channel usage information with each other and make a more accurate decision on the state of this channel. Moreover, the packet collision between SUs and PUs can significantly decrease, since the cooperative sensing method can detect the activity of PUs reoccupy their channels with a large probability.

(ii) We propose a hybrid transmission model combining Overlay and Underlay models to fully utilize the available idle spectrum. Each SU can opportunistically access the unoccupied PU channel or underlay part of its signal into the portion of the channel occupied by the PU depending on the data queue state and sensing result or decide whether or not to access a busy channel to harvest RF energy given its energy queue. Extensive simulations show that our proposed hybrid transmission model can improve the efficiency of spectrum usage and the energy harvesting efficiency of SUs.

(iii) With the aim of eliminating the channel competition among SUs and reducing their average waiting time and also decreasing their spectrum handoff delay, we propose a competitive set based channel selection policy. In our proposed policy, the SUs who form a competitive set in the same channel randomly obtain integer labels from zero. The SU who obtains the zero label has the right to use this channel. In particular, several SUs can obtain multiple labels in different competitive sets. Therefore, the average waiting time that SUs spend on switching to other idle channels or still staying on this channel for data transmission or accessing a busy channel for energy harvesting is lower compared with the random selection policy. Simulation results show that the proposed channel selection policy is simple and effective on reducing the collisions among SUs.

The rest of this paper is organized as follows. The system model is described in Section 2, and the cooperative spectrum 


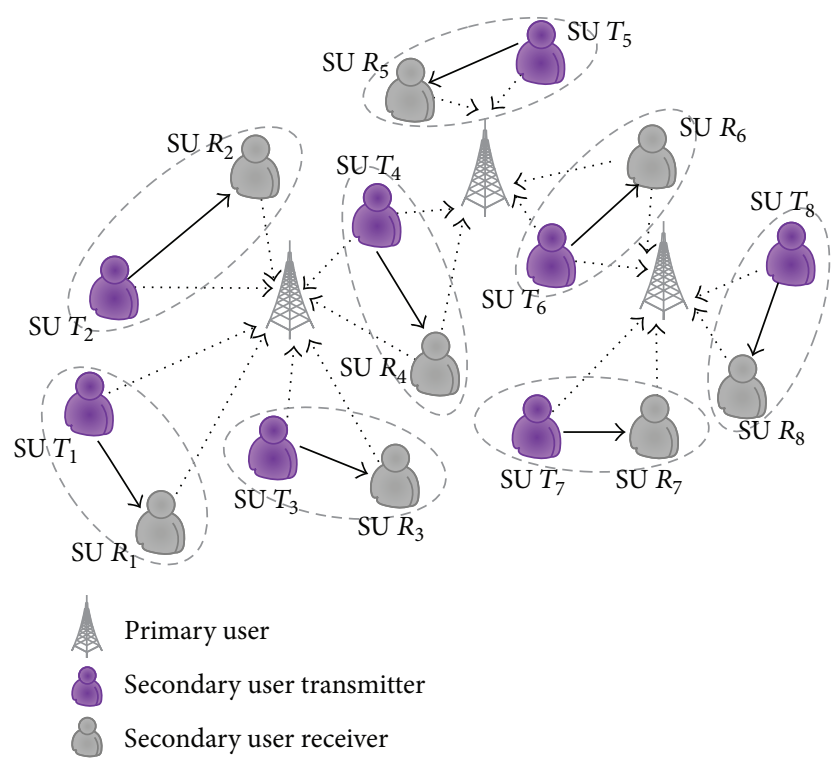

FIGURE 1: A scenario of the multi-SU and multi-PU CR network.

sensing method is given in Section 3. In Section 4, we present the hybrid transmission model and channel selection policy and then analyze the performance in terms of average throughput, average waiting time, and energy harvesting efficiency of SUs' three aspects. The simulation results are listed in Section 5. Finally, we conclude our work in Section 6.

\section{System Model}

2.1. Multi-SU and Multi-PU CR Networks. We consider a multi-SU and multi-PU CR network with $M$ PUs and $N$ pairs of SU, as depicted in Figure 1, where each PU is allocated a licensed channel (which we call "PU channel"). Similar to [13-15], the traffic of each channel is modeled as a two-state continuous-time Markov process: the spectrum is occupied by the PU (busy state) and the spectrum is not occupied by the PU (idle state). For the PUs, these two states are referred to as ON and OFF states, respectively. Each SU transmitter and its corresponding SU receiver are within each other's transmission range. Therefore, the existence of a communication between two SUs depends not only on the distance between them, but also on the time-varying activities of the PUs. As illustrated in Figure 1, we consider the scenario that several SUs may access the same channel, and one SU may have more than one channel for selection.

As these PUs are in the interference range of some SUs, the channel power gains from the PU transmitter to the PU receiver, $\mathrm{SU}$ transmitter to the $\mathrm{SU}$ receiver, $\mathrm{PU}$ transmitter to the SU receiver, and SU transmitter to the PU receiver are denoted by $G_{p p}, G_{s s}, G_{p s}$, and $G_{s p}$, respectively. We employ the model $G_{i j}=k d_{i j}^{-\alpha}$ for the channel gain between the $i$ th transmitter and the $j$ th receiver, where $k$ is an attenuation factor that represents power variation caused by path loss, $d_{i j}$ denotes the distance between them, and $\alpha$ is the path loss [27]. We assume that the channel power gains and the channel state information (CSI) are known to each SU, and SUs can obtain the channel availability after spectrum sensing.

2.2. Energy Harvesting Technology. RF energy signal can not only propagate over a distance but also broadcast in all directions [22]. However, due to the uncertainty of location, fading, and environmental conditions, the energy supplied from RF energy may not guarantee QoS in wireless applications. To ensure the static and stable energy, the $\mathrm{RF}$ energy signal is transformed to a DC voltage and then stored into a rechargeable battery [23]. It is reasonable to define the effective zone of the energy harvesting, since the propagation energy drops off rapidly with the distance increases. We assume that each SU can only obtain the RF energy signal from the channels that it can sense. Each SU can harvest RF energy from the busy channels occupied by PUs and store the energy in a rechargeable battery when its transmitter is equipped with an energy harvesting device, and the maximum size of battery is $E_{\max }$. In this paper, the rechargeable battery is modeled by an ideal linear model [28], where the changes in the energy stored are linearly related to the amounts of energy harvested or spent. Since the increased energy harvested from PU channels can be utilized for channel sensing and data transmission, the working time of SUs will be extended.

\section{Cooperative Spectrum Sensing}

Spectrum sensing is the basis of the DSA mechanism. Furthermore, sensing errors will affect the performance of SUs transmission and cause packet collision between SUs and PUs. In this section, we describe our cooperative spectrum sensing method.

3.1. Energy Based Spectrum Sensing. Spectrum sensing has to be performed before data transmission to detect the channel availability. Many signal techniques have been used for the SUs to sense the activity of the PUs [13]. The energy detection method not only implements simply but also represents intuitively the proportion of the busy channels. Therefore, the energy detection method is accurate and optimal when the SUs have little or no prior knowledge of the PU signal [29], and we consider it as the spectrum sensing algorithm in our proposed policy. The aim of spectrum sensing is to sense the existence of signal in licensed spectrum. Thus, under the two hypotheses, the signal can be expressed as

$$
\begin{aligned}
& H_{0}: x(t)=n(t), \\
& H_{1}: x(t)=s(t)+n(t),
\end{aligned}
$$

where $n(t)$ is an Additive White Gaussian Noise (AWGN) and $s(t)$ is the signal of PU in target channel. $H_{0}$ and $H_{1}$ are the two hypotheses of nonexistence or existence of $s(t)$. From [30], we have known that the probability of detection can be denoted by $P_{d}$ with a fixed SNR $\gamma$ in an AWGN channel, and it can be written as

$$
P_{d}(\gamma, \tau, \lambda)=Q\left(\left(\frac{\lambda}{\sigma^{2}}-\gamma-1\right) \sqrt{\frac{\tau f_{s}}{2 \gamma+1}}\right),
$$




\begin{tabular}{|c|c|c|c|}
\hline \multirow{2}{*}{$\begin{array}{l}\text { Channel } \\
\text { sensing }\end{array}$} & \multirow{2}{*}{$\begin{array}{l}\text { Exchange } \\
\text { information }\end{array}$} & \multirow{2}{*}{ Decision } & Data transmission \\
\hline & & & Energy harvesting \\
\hline
\end{tabular}

FIgURE 2: An intuitional illustration of the time-slot structure.

where $\tau$ is the sensing duration, $\lambda$ is the sensing threshold, $f_{s}$ is the sampling frequency, $\sigma^{2}$ is the variance of the AWGN, and $\mathbb{Q}(x)$ is the tail probability of the normal distribution. Under imperfect sensing, there are two types of sensing errors: miss detection and false alarm. A false alarm error occurs when the SU observes the channel is busy whereas it is actually idle, and a miss detection error occurs when the SU observes the channel is idle whereas it is actually busy. Hence, the false alarm indicates the waste of spectrum access opportunity, whereas the miss detection imposes on the potential interference to PUs. The false alarm probability $P_{f}$ and miss detection probability $P_{m}$ can be expressed as [31]

$$
\begin{gathered}
P_{f}(\tau, \lambda)=Q\left(\left(\frac{\lambda}{\sigma^{2}}-1\right) \sqrt{\tau f_{s}}\right), \\
P_{m}(\gamma, \tau, \lambda)=1-Q\left(\frac{\lambda / \sigma^{2}-(1+\gamma)}{(1+\gamma) \sqrt{2 / \tau f_{s}}}\right),
\end{gathered}
$$

where $P_{f}$ and $P_{m}$ are related to the threshold $\lambda$ and the sensing time $\tau$. Furthermore, $P_{m}$ is also a function of SNR.

3.2. Cooperative Spectrum Sensing. Due to the effects of multipath fading, inside buildings with high penetration loss and local interference, the probability of miss detection and false alarm will be increased under the conventional noncooperative spectrum sensing method. This phenomenon will lead to packet collision between SUs and PUs in multiSU and multi-PU CR networks. In order to deal with this problem, cooperative spectrum sensing has been adopted in some studies $[15,17,23]$. We focus on how to collect the spectrum sensing data of SUs for cooperative sensing and combine these sensing results to produce the final decision in this paper.

As illustrated in Figure 2, we suppose the multi-SU and multi-PU CR network with time slotted (TS), that is, one TS consists of two phases, which are the channel sensing phase (CS) and the data transmission (DT) or energy harvesting (EH) phase, respectively. In the first phase, SUs sense the PU channels to detect the activity of the PUs and exchange the channel usage information with other SUs. Then, each SU will combine its sensing results with others'. At last, two or more of the same results are considered to be the final decision of this channel. In particular, the channel will be redetected until a decision is made when a channel is detected by four SUs, and two of them believe that the channel is idle while the other two are just the opposite. The sensing result is considered to be the final decision when a channel is only detected by one SU. In the next phase, the SU executes RF energy harvesting or data transmission based on the final decision. Similar to [32,33], we suppose that sensing duration

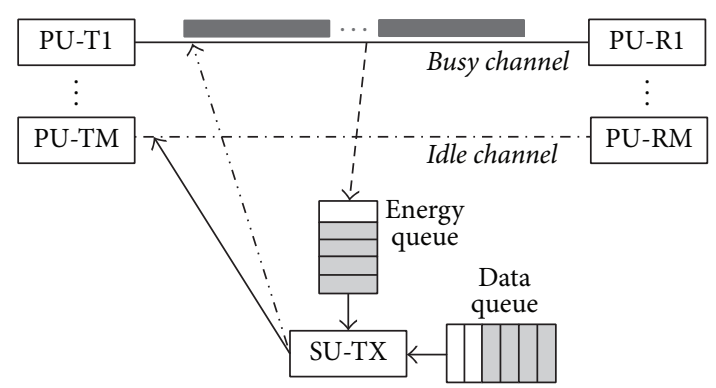

FIgURe 3: An illustration of the hybrid transmission model.

is small, compared with the PU channel traffic state cycle, so that the PU channel traffic state can be considered unchanged during sensing phases.

\section{Channel Selection Policy}

In this section, we first propose a hybrid transmission model for single SU and secondly present a channel selection policy for multi-SU based on the competitive set to alleviate the channel competition among SUs.

4.1. Hybrid Transmission Model. As shown in Figure 3, the arriving data is buffered in the data queue of the SU transmitter, $Q_{D i}, i=1,2,3, \ldots, N$. The maximum capacity of the data queue is $Q_{\max }$. As mentioned before, the RF energy is stored in the energy queue, $Q_{E i}, i=1,2,3, \ldots, N$, whose maximum size is denoted as $E_{\max }$.

At the beginning, when the data arrives at $i$ th SU transmitter, its data queue and energy queue can be represented as $Q_{D i} \neq \varnothing, Q_{E i}=E_{\max }$; then the $S U$ can perform data transmission when idle channels are sensed. Let $E(s)$ be the sensing outcome of $i$ th SU, and we define $\lambda_{O}$ and $\lambda_{U}$ be the Overlay and the Underlay model energy threshold, respectively. If the channel signal energy is sensed below the Overlay model energy threshold, that is, $E(s)<\lambda_{\mathrm{O}}$, the $\mathrm{SU}$ will transmit data with a higher power from its data queue in the Overlay model. However, if the channel signal energy is above the Overlay model energy threshold but below the Underlay model energy threshold, that is, $\lambda_{O}<E(s)<\lambda_{U}$, it means that the PU does not fully occupy this channel, and the SU can access it with PU at the same time by reducing its transmission power as long as it does not interfere in the PU transmission, that is, Underlay transmission model.

To make use of the hybrid transmission model, each SU transmitter is assumed to have perfect knowledge of the CSI. For different channels, their capacity and utilization rate are different. Based on the sensing result of channels, each SU calculates the statistical Overlay and Underlay model energy threshold and updates them according to the two types of sensing errors, that is, miss detection and false alarm. When the data arrives at a SU transmitter, it compares the current channel sensing results with the knowledge of CSI to obtain the occupancy of PU. The SU estimates the power of the PU based on the transmission distance and antenna gain when the PU does not fully occupy this channel [34]. For 


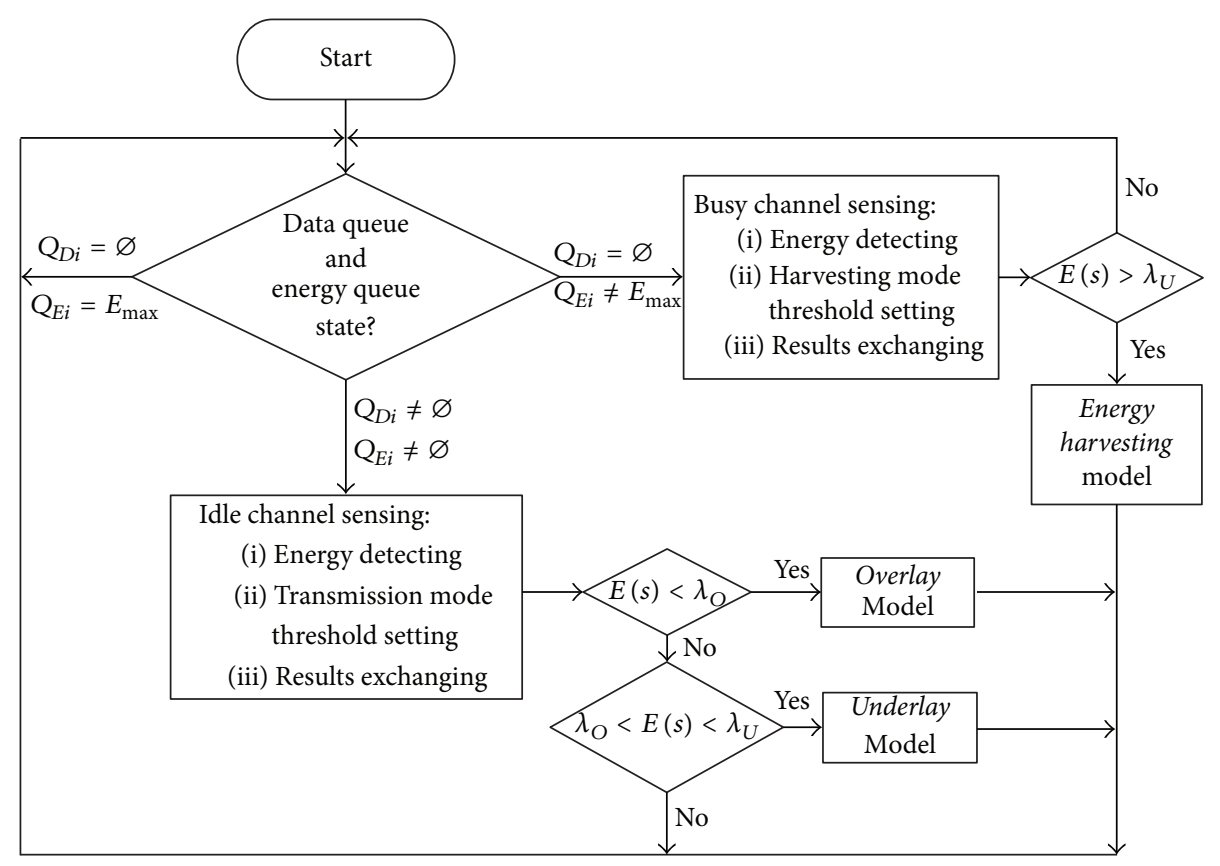

FIGURE 4: An illustration of the process of selecting the transmission models for each SU.

the Overlay model, there is no limitation on the transmit power of SUs, and they can transmit data with the initial power. Nevertheless, due to the interference caused by SU towards PU, the SUs need to decrease the transmit power, change the modulation type, and adjust the encoded mode to afford a suitable SNR to accommodate the variation of current channel in the Underlay model.

When the data queue of $i$ th SU is empty and its energy is used in the previous time-slot, that is, $Q_{D i}=\varnothing, Q_{E i} \neq$ $E_{\max }$, the SU can harvest RF energy from busy channels for increasing energy reserves. Hence, if a channel is sensed above the Underlay model energy threshold, the SU may implement energy harvesting from it.

In our proposed hybrid transmission model, each SU can determine to implement either the data transmission or the energy harvesting depending on the state of data queue and energy queue. Based on the spectrum sensing results and Overlay/Underlay model energy thresholds, each SU can not only access a channel alone or with the PU simultaneously for data transmission but also harvest the RF energy from the PU occupied channels. The SU decides whether or not to stay in the current channel or switch to a new channel for data transmission or a busy channel for energy harvesting after sensing channels in the next CS phase. The process of selecting the transmission models for each $\mathrm{SU}$ is presented in Figure 4.

4.2. Overview of Channel Selection Policy. As the mentioned hybrid transmission model, each SU can implement either the data transmission in an idle channel or the energy harvesting from a busy channel. However, for the multi-SU and multi-PU CR network, one of the great challenges of implementing multi-SU channel access successfully is the problem of competition among SUs. We explain the details of the proposed channel selection policy.

4.2.1. Channel Selection Policy for Data Transmission. The SU transmitter sends a RTS packet on the channel to its corresponding SU receiver if an idle channel is detected. Then the SU receiver replies with a CTS packet in the same channel. Notice that the RTS/CTS collision may occur when more than one pair of SUs contends the same target idle channel for data transmissions. Hence, different from the conventional way, the SU pair does not access the idle channel immediately when the CTS packet is successfully received by the SU transmitter. Those SUs who receive CTS packet form a competitive set, $S_{I i}, i=1,2,3, \ldots, M$, which means that these SUs are competing to access this PU channel. Supposing that the size of $S_{I i}$ is $W$, we randomly assign them integer labels from zero to $W-1$. The SU who obtains the zero label can transmit data in the DT phase. In particular, for the SUs who can sense more than one channel, they can compete for multiple idle channels and obtain multiple labels when the data arrives at their data queue. Furthermore, the SU can access the corresponding channel for data transmission as long as it can obtain the zero label in one competitive set. Similarly, when the channel can only be accessed in the Underlay model for data transmission, those SUs who receive the CTS packet will form a competitive set, $S_{U i}, i=$ $1,2,3, \ldots, M$.

The SU that transmits data in the previous time-slot will keep data transmission until the channel state is changed when the sensing outcome of the current channel is $E(s)<$ $\lambda_{O}$ in the next CS phase. All the label values of other SUs are in the same competitive set minus one when the SU withdraws from the current channel. Therefore, the SU whose 


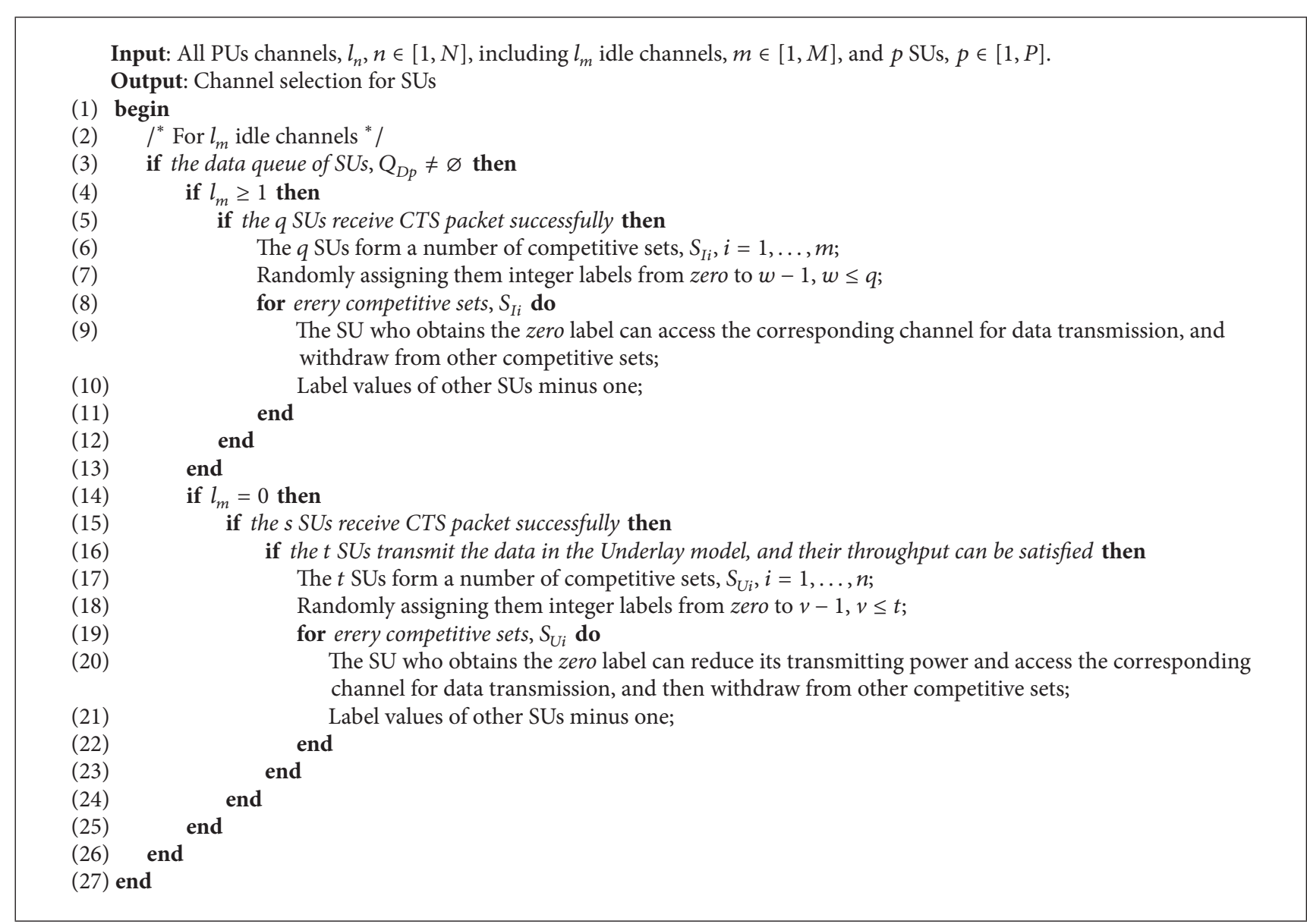

Algorithm 1: The channel selection policy for data transmission.

label value is subtracted to be zero can access this channel. If the present channel is detected satisfy $\lambda_{O}<E(s)<$ $\lambda_{U}$ in the next CS phase; that is, the PU is not completely occupied in this channel for data transmission, the SU reduces its transmission power to satisfy the interference power constraint of $\mathrm{PU}$ that it can continue to transmit data in the Underlay model, and their corresponding competitive sets will remain in use. However, the transmission of SU will cause interference to the communication of PU if the $E(s)>\lambda_{U}$. Then the data transmission of SU will be stopped in the next DT phase, and the competitive set of this channel will be dissolved. The algorithm of our channel selection strategy for data transmission is presented in Algorithm 1.

We illustrate the process of randomly assigning integer labels by Figure 5. First, four SUs need to access channels for data transmission, and they sense the current channel availability to obtain a list of available channels. Secondly, the SUs who compete for the same channel form a competitive set; that is, the SUs 1, 2, 3 can use the channel A. We randomly assign integer labels from zero for these SUs. Thirdly, the SUs who obtain the zero label can access the channels, and they withdraw from the corresponding competitive sets while the label values of other SUs are minus one. In particular, the SU 2 can use channels A or B. In the next time-slot, the SU 4 can access the channel $\mathrm{B}$.
4.2.2. Channel Selection Policy for Energy Harvesting. The SUs that contend for the same target busy channel form a competitive set, $S_{E j}, j=1,2,3, \ldots, M$, which means that these SUs are competing to access the $j$ th PU channel for energy harvesting. We also assign them integer labels, and the SU that obtains the zero label can harvest energy in the next $\mathrm{EH}$ phase. Then these SUs withdraw from the competition sets when the data arrives at the data queue of SUs or their energy queue is full. The algorithm of our channel selection policy for energy harvesting is given in Algorithm 2.

In the proposed channel selection policy, the SU receiver sends a Decode packet to its transmitter when the current transmission is complete; that is, the data has been accepted successfully. The Channel-Switching (CSW) flag is set when the SU needs to switch to another channel, and then the SU transmitter and receiver pause their current transmission and perform channel handoff $[35,36]$.

Note that the CSMA/CA protocol also uses the RTS/CTS handshake procedures to ensure that the collision does not occur among users and utilizes the exponential-backoff algorithm to decompose collision; that is, each node performs a random delay $t$ when the collision happens, and $t$ obeys the $T(0 \sim T)$ on the bottom of the exponential distribution. In our proposed channel selection policy, we ensure the usage of idle channels through establishing the competitive sets 


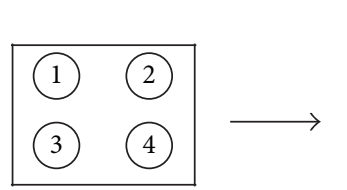

The SUs that need to access channels.

\begin{tabular}{|c|c|}
\hline User & $\begin{array}{c}\text { Available } \\
\text { channel }\end{array}$ \\
\hline (1) & A, C \\
\hline 2 & A, B \\
\hline (3) & A \\
\hline (4) & B \\
\hline
\end{tabular}

The SUs sense the usage of current channels and obtain the list of available channels.

$\longrightarrow$\begin{tabular}{|c|c|c|}
\hline User & $\begin{array}{c}\text { Available } \\
\text { channel }\end{array}$ & Label \\
\hline (1) & A, C & 1,0 \\
\hline (2) & A, B & 0,0 \\
\hline (3) & A & 2 \\
\hline (4) & B & 1 \\
\hline
\end{tabular}

We randomly assign integer labels for each SU.

Next time-slot.

The SU who obtains the zero label can access one channel while the label values of other SUs minus one.

FigURE 5: An example of randomly assigning integer labels.

Input: All PUs channels, $l_{n}, n \in[1, N]$, including $l_{m}$ idle channels, $m \in[1, M]$, and $p$ SUs, $p \in[1, P]$.

Output: Channel selection for SUs

(1) begin

(2) $/{ }^{*}$ For $l_{n-m}$ busy channels $* /$

(3) if the data queue of $S U s, Q_{D p}=\varnothing$ then

(4) if the energy queue of $S U s, Q_{D p^{\prime}} \neq Q_{\max }$ then

(5) The $p^{\prime}$ SUs form a number of competitive sets, $S_{E i}, i=1, \ldots, n$;

(6) Randomly assigning them integer labels from zero to $u-1, u \leq p^{\prime}$;

(7) for erery competitive sets, $S_{E i}$ do

(8) The SU who obtains the zero laber can access the corresponding busy channel for energy harvesting in the next $\mathrm{EH}$ phase, and withdraw from other competitive sets;

(9) Label values of other SUs minus one;

$(11)$

$(12)$ end end

end

(13) end

Algorithm 2: The channel selection policy for energy harvesting.

and randomly assigning the integer labels when the collision occurs. Moreover, in order to reduce the average waiting time of SUs, the SUs who access channels withdraw from their competitive sets while other SUs' label values are minus one.

\subsection{Performance Analysis of the Channel Selection Policy.} In this section, we intend to illustrate the spectrum usage performance of our proposed policy in terms of average throughput, average waiting time, and energy harvesting efficiency of SUs' three aspects.
4.3.1. Average Throughput of SUs. In our proposed channel selection policy, SU can transmit data in the Overlay or Underlay model. The service rate of each SU in the hybrid model is described as $R_{h}=R_{o}+R_{u}$, and $R_{o}$ can be denoted by $R_{o}^{0}, R_{o}^{1}, R_{o}^{0^{\prime}}$, and $R_{o}^{1^{\prime}}$ in the Overlay model [37]

$$
\begin{aligned}
& R_{o}^{0}=B \log _{2}\left(1+g_{s} P_{s}^{o}\right), \\
& R_{o}^{1}=0,
\end{aligned}
$$




$$
\begin{aligned}
& R_{o}^{0^{\prime}}=B \log _{2}\left(1+\frac{g_{s} P_{s}^{o}}{g_{p} P_{p}+1}\right), \\
& R_{o}^{1^{\prime}}=0,
\end{aligned}
$$

where $R_{o}^{0}$ represents that the PU does not occupy the channel. In contrast, $R_{o}^{1}$ represents that the spectrum is being occupied by PU. $R_{o}^{0^{\prime}}$ and $R_{o}^{1^{\prime}}$ are the service rate of each SU under false alarm and miss detection, respectively. Similarly, the $R_{u}$ can be denoted by $R_{u}^{0}, R_{u}^{1}$ in the Underlay model

$$
\begin{aligned}
& R_{u}^{0}=B \log _{2}\left(1+g_{s} P_{s}^{u}\right), \\
& R_{u}^{1}=B \log _{2}\left(1+\frac{g_{s} P_{s}^{u}}{g_{p} P_{p}+1}\right) .
\end{aligned}
$$

The throughput of SU can be described in terms of the outage as [38]

$$
T=1-p_{\text {out }},
$$

where $p_{\text {out }}$ is the outage probability. The throughput in channel selection policy, $T_{h}$, is comprised of $T_{o}$ and $T_{u}$, respectively. $T_{o}$ is given by

$$
T_{o}=p_{i}\left(1-p_{f}\right)\left(1-p_{\text {out }}^{o}\right)+\left(1-p_{i}\right) p_{f}\left(1-p_{\text {out }}^{o^{\prime}}\right),
$$

where $p_{i}$ is the channel idle probability. Since the SU transmission will cause interference to PU Under false alarm, $p_{\text {out }}^{o}$ and $p_{\text {out }}^{o^{\prime}}$ can be described as

$$
\begin{aligned}
& p_{\text {out }}^{o}=\operatorname{Pr}\left[R_{o}^{0}<R_{s}\right], \\
& p_{\text {out }}^{o^{\prime}}=\operatorname{Pr}\left[R_{o}^{0^{\prime}}<R_{s}\right],
\end{aligned}
$$

where $R_{s}$ is the required service rate of SU. Correspondingly, we can obtain $T_{u}, p_{\text {out }}^{u}$, and $p_{\text {out }}^{u^{\prime}}$ as follows:

$$
\begin{aligned}
T_{u} & =p_{i}\left(1-p_{f}\right)\left(1-p_{\text {out }}^{u}\right)+\left(1-p_{i}\right) p_{f}\left(1-p_{\text {out }}^{u^{\prime}}\right), \\
p_{\text {out }}^{u} & =\operatorname{Pr}\left[R_{u}^{0}<R_{s}\right], \\
p_{\text {out }}^{u^{\prime}} & =\operatorname{Pr}\left[R_{u}^{1}<R_{s}\right] .
\end{aligned}
$$

4.3.2. Average Waiting Time of SUs. Here, we calculate the time elapsed between each SU which receives the RTS signal and implements data transmission, and this elapsed time can reflect the performance of the competitive set. The average waiting time of SUs will be longer than the conventional random access policy if the design of the competitive set is not reasonable. Thus, the average waiting time of SUs, $T_{w}$, can be described as

$$
T_{w}=T_{t}-T_{\mathrm{RTS}},
$$

where $T_{t}$ and $T_{\mathrm{RTS}}$ are the time-slots that the SU transmits data to and receives the RTS signal from, respectively.
TABLE 1: Simulation parameters settings.

\begin{tabular}{lc}
\hline Parameter & Value \\
\hline$P_{i}$ & 0.8 \\
$\lambda_{o}$ & 0.3 \\
$\lambda_{u}$ & 0.7 \\
$E_{\max }$ & 15 \\
$Q_{\max }$ & 20 \\
$R_{s}$ & $3 \mathrm{bps}$ \\
$P_{p}$ & $15 \mathrm{~dB}$ \\
Time-slot & $2 \mathrm{~ms}$ \\
RTS packets length & $250 \mathrm{bit}$ \\
CTS packets length & $220 \mathrm{bit}$ \\
\hline
\end{tabular}

4.3.3. Energy Harvesting Efficiency. We use $e_{h}$ to express the packets of energy that can be harvested by SUs from busy channels, and it follows Poisson distribution. The energy consumed by SU for data transmission and spectrum sensing are $e_{t}$ and $e_{s}$, respectively. We assume that $e_{c}$ represents another energy consumption on circuit and $e_{r}^{t}$ denotes the residual energy at the time-slot $t$. Therefore, the energy harvesting efficiency is described in terms of the residual energy in the next time-slot

$$
e_{r}^{t+1}=\min \left[e_{r}^{t}+e_{h}-\left(e_{t}+e_{s}+e_{c}\right), E_{\max }\right] .
$$

\section{Simulations}

In this section, we will provide numerical results to demonstrate the performance of the proposed cooperative sensing method and channel selection policy in terms of probability of false alarm, average throughput, average waiting time, and energy harvesting efficiency of SUs. Table 1 shows the parameter settings of our simulations, and some parameters are valued based on the previous works on CR networks. We consider a multi-SU and multi-PU CR network with 20 PUs, 20 available PU channels, and 25 pairs of SUs. In particular, several SUs may access the same channel, and one SU may have more than one channel for selection. We set all the spectrum bandwidth to be the same, and the packets lengths of SUs and PUs are fixed in the simulations. However, the interference limitation of PUs is different. We let the path loss constant $\alpha=2$ and attenuation factor $k=0.5$, respectively, according to the empirical values to compute the channel gain. Moreover, the white Gaussian noise is $8 \times 10^{-15}$.

5.1. Performance for Probability of False Alarm. Figure 6 illustrates the probability of false alarm in cooperative sensing and conventional noncooperative sensing method under different numbers of SUs. As shown in the figure, the probability of false alarm decreases with the increase of detection probability. For the conventional noncooperative sensing method, there is no interaction on the detection results among multiple SUs. Hence, the increase of users has no impact on the probability of false alarm. For a fixed detection probability, cooperative sensing method can achieve higher detection accuracy. As described in Section 3.2, two or more 


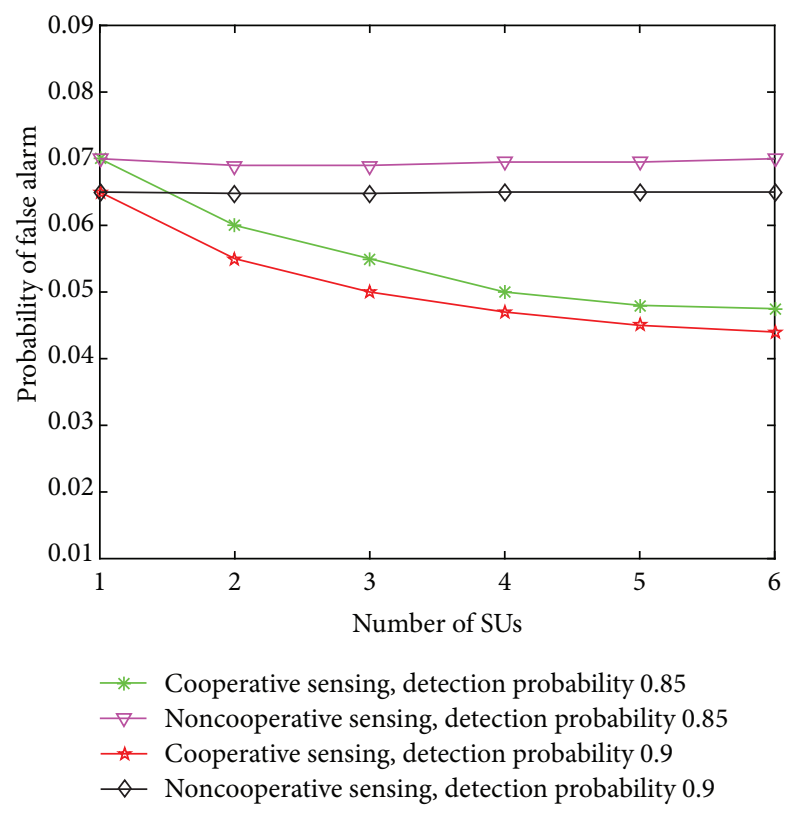

FIGURE 6: The probability of false alarm in cooperative sensing and noncooperative sensing methods under different numbers of SUs.

of the same results are considered to be the final decision of the target channel. Therefore, the probability of false alarm decreases significantly when the cooperative SUs are 2 or 3 . Furthermore, the probability of false alarm where the detection probability is 0.85 decreases faster when the number of SUs changes from 3 to 4 . Thus, the cooperative sensing method can improve the accuracy of channel sensing in the case of low detection rate. However, more than 4 cooperative SUs have little effect on the probability of false alarm.

5.2. Performance for Average Throughput of SUs. Figure 7 shows the average throughput of SUs in the following transmission models: our proposed hybrid model, existing hybrid model [39], Overlay-only model, and Underlay-only model under different numbers of busy channels. As can be seen from the figure that the average throughput of SUs decreases in the Overlay-only model with the number of busy channels increase. It is due to the fact that the high percentage of busy channels restricts SUs from transmission in the Overlayonly model. However, there is a little impact on the average throughput of SUs, since the SUs can coexist with PUs in the Underlay-only model. It can be observed from the figure that the hybrid model transmission outperforms the Overlay-only and Underlay-only model alone. Furthermore, we can see that our proposed hybrid model can achieve higher throughput compared with the existing hybrid model. The reason of this observation can be explained as follows. With the decrease of available idle channels, the opportunities of SUs for data transmission become less. The collision among SUs becomes more intense, since the existing hybrid model is only based on the number of SUs and access probability. However, the concept of competitive set can improve the utilization of the limited idle channels.

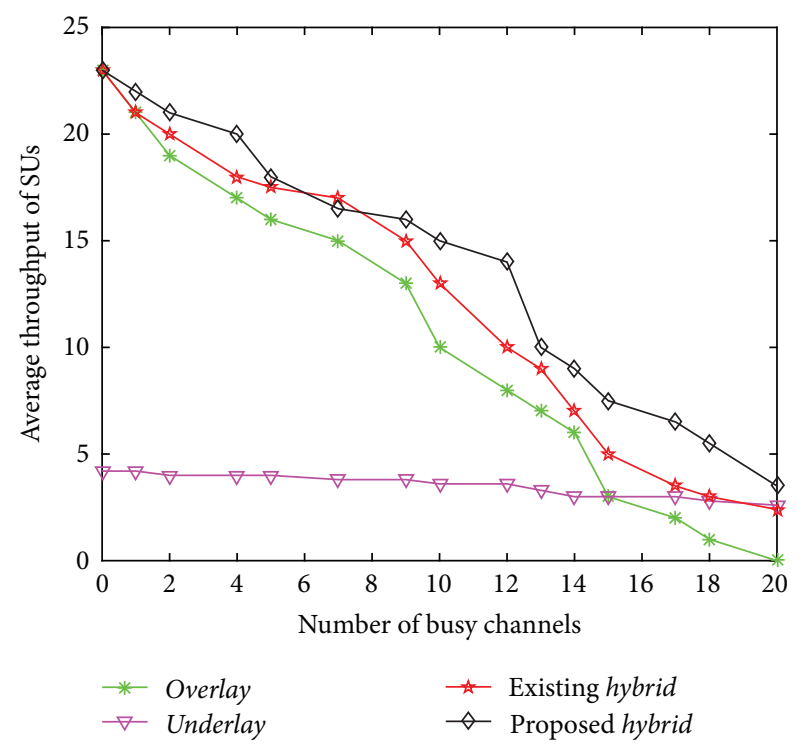

FIGURE 7: The effect of transmission models on the average throughput of SUs under different numbers of busy channels.

5.3. Performance for Average Waiting Time of SUs. Figure 8 shows the average waiting time of SUs in four different models under different numbers of busy channels. It is clear that the average waiting time of SUs greatly increases in the Overlayonly model with the decrease of available idle channels. In contrast, the average waiting time is not significantly increased in the Underlay-only model, since the number of available idle channels has little effect on the data transmission of SUs. The different interference threshold constraint of PUs so that the SUs cannot access some channels is in the Underlay-only model, which results in the consequence that some SUs need to wait for a long time to access channels. The average waiting time of SUs of our proposed hybrid model is lower than the existing hybrid model but higher than the Underlay-only model when lots of channels are occupied by PUs. The explanation for this observation is as follows. As described in Section 4, SUs can continue to transmit data in the Underlay model when the PU accesses its idle channel, and their current competitive sets will remain in use. Furthermore, the SUs that compete more than one channels can wait for accessing opportunities in other competitive sets when the current channel cannot be accessed. Therefore, the average waiting time of SUs will be reduced in our proposed hybrid model. However, since the hybrid model will give priority to whether the channel can be accessed in the Overlay model when the idle channels become less, the average waiting time of SUs in the Underlay-only model is lower than ours.

5.4. Performance for Energy Harvesting Efficiency of SUs. Figure 9 shows the average residual energy of SUs in the conventional CR network, existing CR network with energy harvesting [30], and our CR network with energy harvesting versus the simulation time. As shown in the figure, energy harvesting technology can ensure that enough energy is reserved after long time communication. Furthermore, our 


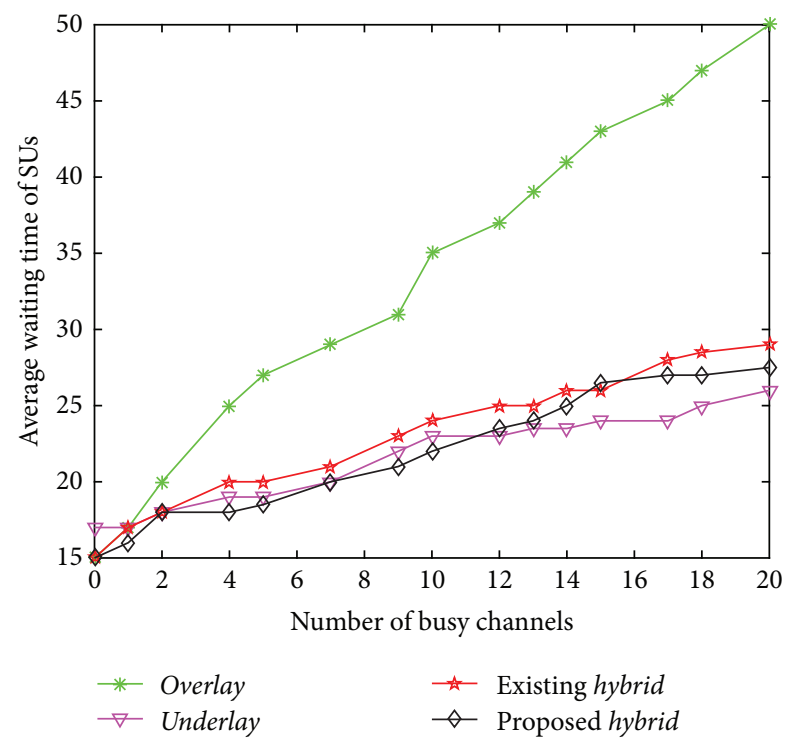

FIGURE 8: The effect of transmission models on the average waiting time of SUs under different numbers of busy channels.

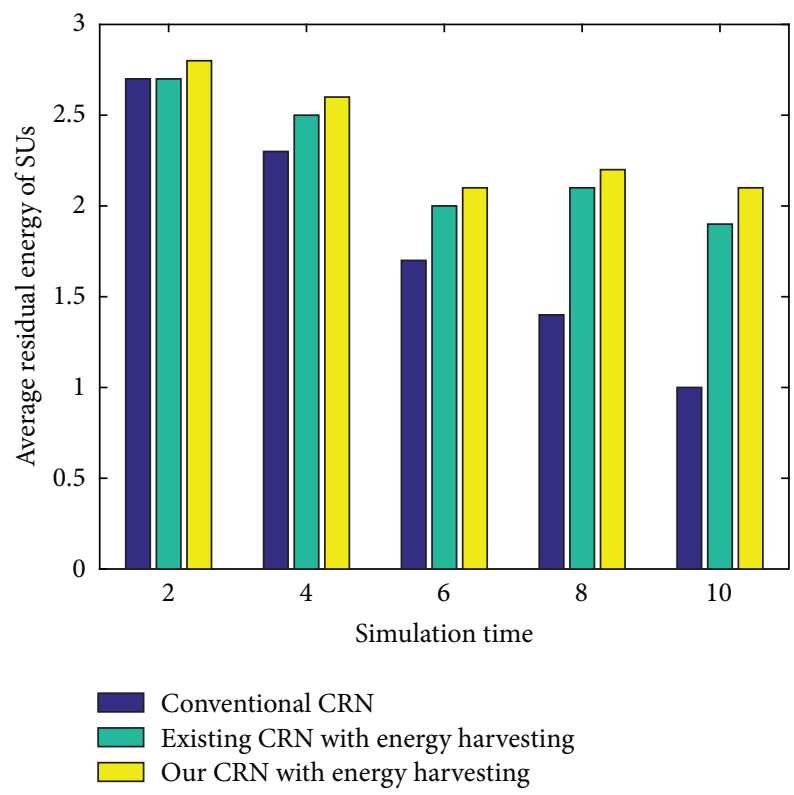

FIGURE 9: The average residual energy of SUs in the conventional CR network, existing CR network with energy harvesting, and our CR network with energy harvesting under different simulation time.

proposed CR network with energy harvesting outperforms the existing CR network with energy harvesting. This is because as described in Section 3, SUs decide to sense the idle channels for data transmission or busy channels for energy harvesting depending on the state of data queue and energy queue. Hence, SUs can spend less energy for sensing channels. Meanwhile, the SUs may have more opportunities to harvest energy, since the concept of competitive set can reduce the collision among multiple SUs competing for the same busy channel.

\section{Conclusion}

In this paper, aiming at solving the problem of spectrum scarcity in IoE environment, we consider a multi-SU and multi-PU cognitive radio network in which the SUs are equipped with the RF energy harvesting capability. In this network, the crucial issues are the channel competition among SUs and the packet collision between SUs and PUs. We adopt the cooperative spectrum sensing method to reduce the probability of sensing errors and alleviate the interference to PUs. In order to solve the problem of channel competition among SUs, we first propose a hybrid transmission model for single SU. Each SU can either implement data transmission in an idle channel or energy harvesting from a busy channel given its data queue and energy queue state and sensing result. Additionally, we present a channel selection policy for multi-SU based on competitive set. Our proposed policy can achieve higher throughput compared with the conventional random policy. Furthermore, the collision will never be detected by themselves and may last for a quite long time when several SUs collide with each other in the conventional random policy. Hence, the channel competition among SUs will largely limit the performance of conventional random policy. While SUs will detect the collision in the CS phase and stop transmission in the next DT/EH phase to avoid longer ineffective transmission in our proposed policy. Simulations show that the proposed cooperative sensing method and channel selection policy outperform previous solutions in terms of probability of false alarm, average throughput, average waiting time, and energy harvesting efficiency of SUs.

\section{Competing Interests}

The authors declare that there are no competing interests regarding the publication of this paper.

\section{Acknowledgments}

This work was supported in part by the National Natural Science Foundation of China, under Grant 61672283, the Funding of Jiangsu Innovation Program for Graduate Education, under Grant KYLX15 0325, the Fundamental Research Funds for the Central Universities, under Grant NS2015094, the Natural Science Foundation of Jiangsu Province, under Grant BK20140835, and the Postdoctoral Foundation of Jiangsu Province, under Grant 1401018B.

\section{References}

[1] A. Al-Fuqaha, M. Guizani, M. Mohammadi, M. Aledhari, and M. Ayyash, "Internet of things: a survey on enabling technologies, protocols, and applications," IEEE Communications Surveys \& Tutorials, vol. 17, no. 4, pp. 2347-2376, 2015.

[2] J. Jin, J. Gubbi, S. Marusic, and M. Palaniswami, "An information framework for creating a smart city through internet of things," IEEE Internet of Things Journal, vol. 1, no. 2, pp. 112-121, 2014.

[3] C. Zhu, V. C. M. Leung, L. Shu, and E. C. H. Ngai, "Green internet of things for smart world," IEEE Access, vol. 3, pp. 21512162, 2015. 
[4] Z. Sheng, C. Mahapatra, C. Zhu, and V. C. M. Leung, "Recent advances in industrial wireless sensor networks toward efficient management in IoT," IEEE Access, vol. 3, pp. 622-637, 2015.

[5] Z. Sheng, C. Zhu, and V. C. M. Leung, "Surfing the internetof-things: lightweight access and control of wireless sensor networks using industrial low power protocols," EAI Endorsed Transactions on Industrial Networks and Intelligent Systems, vol. 14, no. 1, article e2, pp. 1-11, 2014.

[6] W. Li, C. Zhu, V. C. M. Leung, L. T. Yang, and Y. Ma, "Performance comparison of cognitive radio sensor networks for industrial IoT with different deployment patterns," IEEE Systems Journal, 2015.

[7] W. Li, V. Leung, C. Zhu, and Y. Ma, "Scheduling and routing methods for cognitive radio sensor networks in regular topology," Wireless Communications and Mobile Computing, vol. 16, no. 1, pp. 47-58, 2016.

[8] J. Li, H. Zhao, J. Wei et al., "Sender-jump receiver-wait: a blind rendezvous algorithm for distributed cognitive radio networks," in Proceedings of the IEEE International Symposium on Personal, Indoor and Mobile Radio Communications (PIMRC '16), Valencia, Spain, September 2016.

[9] Federal Comunications Commission, "Unlicensed operation in the TV broadcast bands," Rep. ET Docket no. 08-260, 2008.

[10] Y. C. Liang, K. C. Chen, G. Y. Li, and P. Mahonen, "Cognitive radio networking and communications: an overview," IEEE Transactions on Vehicular Technology, vol. 60, no. 7, pp. 33863407, 2011.

[11] E. Z. Tragos, S. Zeadally, A. G. Fragkiadakis, and V. A. Siris, "Spectrum assignment in cognitive radio networks: a comprehensive survey," IEEE Communications Surveys \& Tutorials, vol. 15, no. 3, pp. 1108-1135, 2013.

[12] X. Zhai, L. Zheng, and C. W. Tan, "Energy-infeasibility tradeoff in cognitive radio networks: price-driven spectrum access algorithms," IEEE Journal on Selected Areas in Communications, vol. 32, no. 3, pp. 528-538, 2014.

[13] Y. Yilmaz, Z. Guo, and X. Wang, "Sequential joint spectrum sensing and channel estimation for dynamic spectrum access," IEEE Journal on Selected Areas in Communications, vol. 32, no. 11, pp. 2000-2012, 2014.

[14] N. Khambekar, C. M. Spooner, and V. Chaudhary, "On improving serviceability with quantified dynamic spectrum access," in Proceedings of the IEEE International Symposium on Dynamic Spectrum Access Networks (DySPAN '14), pp. 553-564, McLean, Va, USA, April 2014.

[15] T. M. C. Chu, H. Phan, and H. J. Zepernick, "Hybrid interweaveunderlay spectrum access for cognitive cooperative radio networks," IEEE Transactions on Communications, vol. 62, no. 7, pp. 2183-2197, 2014.

[16] V. Chakravarthy, X. Li, R. Zhou, Z. Wu, and M. Temple, "Novel overlay/underlay cognitive radio waveforms using sd-smse framework to enhance spectrum efficiency-part II: analysis in fading channels," IEEE Transactions on Communications, vol. 58, no. 6, pp. 1868-1876, 2010.

[17] A. K. Karmokar, S. Senthuran, and A. Anpalagan, "Physical layer-optimal and cross-layer channel access policies for hybrid overlay-underlay cognitive radio networks," IET Communications, vol. 8, no. 15, pp. 2666-2675, 2014.

[18] J. Zou, H. Xiong, D. Wang, and C. W. Chen, “Optimal power allocation for hybrid overlay/underlay spectrum sharing in multiband cognitive radio networks," IEEE Transactions on Vehicular Technology, vol. 62, no. 4, pp. 1827-1837, 2013.
[19] H. Cho and G. Hwang, "An optimized random channel access policy in cognitive radio networks under packet collision requirement for primary users," IEEE Transactions on Wireless Communications, vol. 12, no. 12, pp. 6382-6391, 2013.

[20] S. Xie and Y. Wang, "Construction of tree network with limited delivery latency in homogeneous wireless sensor networks," Wireless Personal Communications, vol. 78, no. 1, pp. 231-246, 2014.

[21] J. Shen, H. Tan, J. Wang, J. Wang, and S. Lee, "A novel routing protocol providing good transmission reliability in underwater sensor networks," Journal of Internet Technology, vol. 16, no. 1, pp. 171-178, 2015.

[22] S. Sudevalayam and P. Kulkarni, "Energy harvesting sensor nodes: survey and implications," IEEE Communications Surveys \& Tutorials, vol. 13, no. 3, pp. 443-461, 2011.

[23] Pratibha, K. H. Li, and K. C. Teh, "Energy-harvesting cognitive radio systems cooperating for spectrum sensing and utilization," in Proceedings of the IEEE Global Communications Conference (GLOBECOM '15), San Diego, Calif, USA, December 2015.

[24] L. Mohjazi, M. Dianati, G. K. Karagiannidis, S. Muhaidat, and M. Al-Qutayri, "Rf-powered cognitive radio networks: technical challenges and limitations," IEEE Communications Magazine, vol. 53, no. 4, pp. 94-100, 2015.

[25] S. Hu, Y. D. Yao, and Z. Yang, "Cognitive medium access control protocols for secondary users sharing a common channel with time division multiple access primary users," Wireless Communications and Mobile Computing, vol. 14, no. 2, pp. 284296, 2014.

[26] H. A. B. Salameh and M. F. El-Attar, "Cooperative OFDMbased virtual clustering scheme for distributed coordination in cognitive radio networks," IEEE Transactions on Vehicular Technology, vol. 64, no. 8, pp. 3624-3632, 2015.

[27] S. P. Herath and N. Rajatheva, "Analysis of equal gain combining in energy detection for cognitive radio over Nakagami channels," in Proceedings of the IEEE Global Telecommunications Conference (GLOBECOM '08), pp. 1-5, New Orleans, La, USA, November 2008.

[28] X. Lu, P. Wang, D. Niyato, D. I. Kim, and Z. Han, "Wireless networks with RF energy harvesting: a contemporary survey," IEEE Communications Surveys \& Tutorials, vol. 17, no. 2, pp. 757-789, 2015.

[29] S. Wang, Y. Wang, J. P. Coon, and A. Doufexi, "Energy-efficient spectrum sensing and access for cognitive radio networks," IEEE Transactions on Vehicular Technology, vol. 61, no. 2, pp. 906-912, 2012.

[30] S. Park, H. Kim, and D. Hong, "Cognitive radio networks with energy harvesting," IEEE Transactions on Wireless Communications, vol. 12, no. 3, pp. 1386-1397, 2013.

[31] T. Yucek and H. Arslan, "A survey of spectrum sensing algorithms for cognitive radio applications," IEEE Communications Surveys \& Tutorials, vol. 11, no. 1, pp. 116-130, 2009.

[32] W. B. Chien, C. K. Yang, and Y. H. Huang, "Energy-saving cooperative spectrum sensing processor for cognitive radio system," IEEE Transactions on Circuits and Systems I: Regular Papers, vol. 58, no. 4, pp. 711-723, 2011.

[33] Y. Zou, Y. D. Yao, and B. Zheng, "Cooperative relay techniques for cognitive radio systems: spectrum sensing and secondary user transmissions," IEEE Communications Magazine, vol. 50, no. 4, pp. 98-103, 2012.

[34] P. J. Smith, P. A. Dmochowski, H. A. Suraweera, and M. Shafi, "The effects of limited channel knowledge on cognitive radio 
system capacity," IEEE Transactions on Vehicular Technology, vol. 62, no. 2, pp. 927-933, 2013.

[35] B. Wang, Z. Ji, K. J. R. Liu, and T. C. Clancy, "Primaryprioritized markov approach for dynamic spectrum allocation," in Proceedings of the IEEE International Symposium on New Frontiers in Dynamic Spectrum Access Networks (DySPAN '07), pp. 1854-1865, Dublin, Ireland, April 2007.

[36] Y. Song and J. Xie, "ProSpect: a proactive spectrum handoff framework for cognitive radio ad hoc networks without common control channel," IEEE Transactions on Mobile Computing, vol. 11, no. 7, pp. 1127-1139, 2012.

[37] M. G. Khoshkholgh, K. Navaie, and H. Yanikomeroglu, "Access strategies for spectrum sharing in fading environment: overlay, underlay, and mixed," IEEE Transactions on Mobile Computing, vol. 9, no. 12, pp. 1780-1793, 2010.

[38] Y. Wang, P. Ren, F. Gao, and Z. Su, "A hybrid underlay/overlay transmission mode for cognitive radio networks with statistical quality-of-service provisioning," IEEE Transactions on Wireless Communications, vol. 13, no. 3, pp. 1482-1498, 2014.

[39] S. Gmira, A. Kobbane, and E. Sabir, "A new optimal hybrid spectrum access in cognitive radio: overlay-underlay mode," in Proceedings of the International Conference on Wireless Networks and Mobile Communications (WINCOM '15), Marrakech, Morocco, October 2015. 

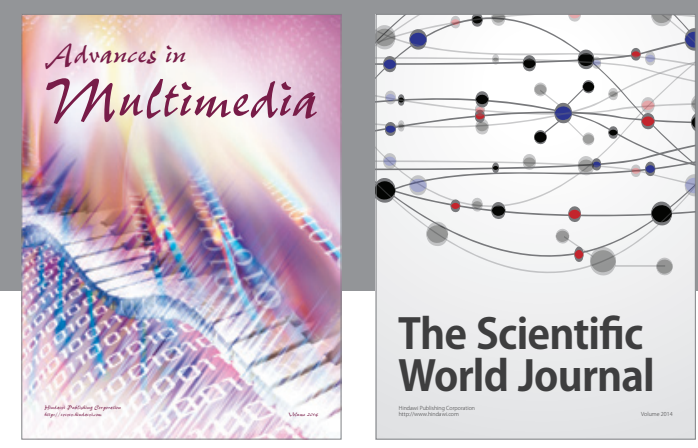

The Scientific World Journal
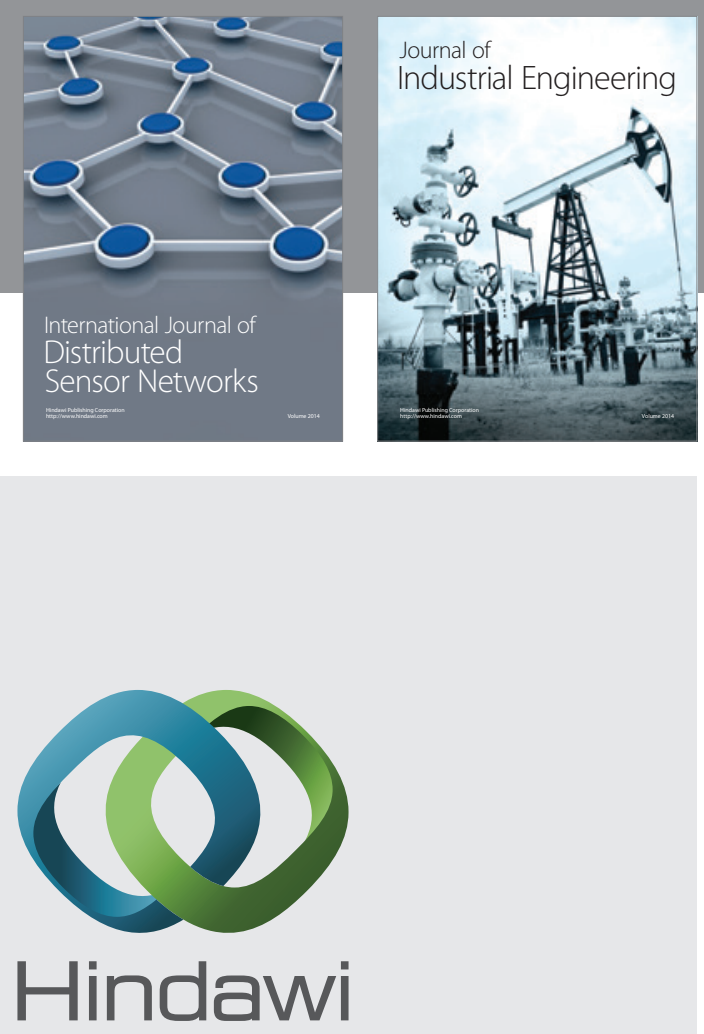

Submit your manuscripts at

http://www.hindawi.com

\section{Computer Networks} and Communications
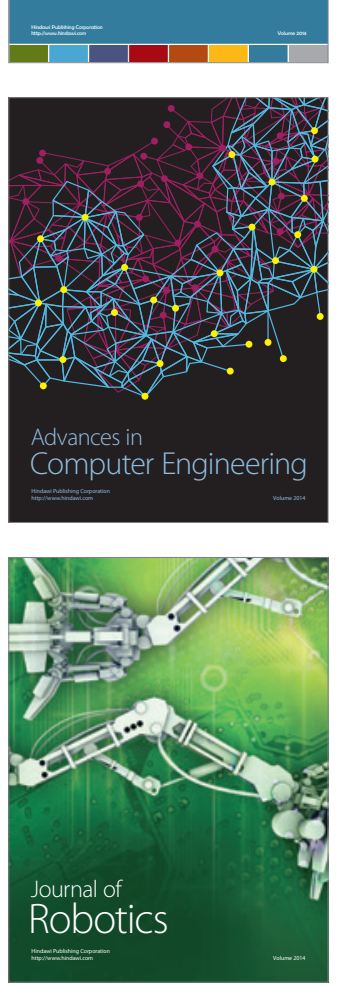
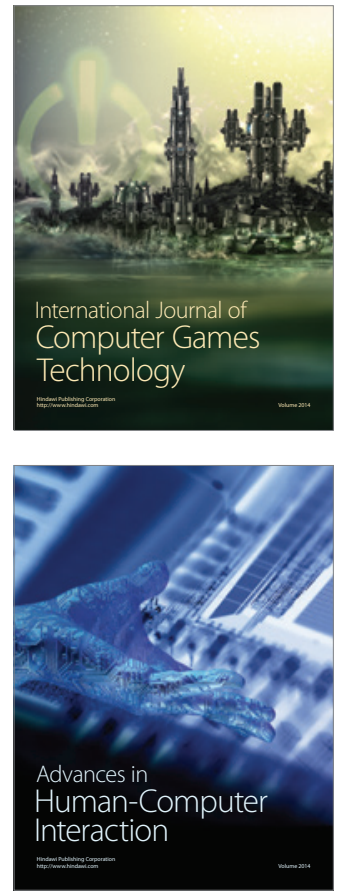
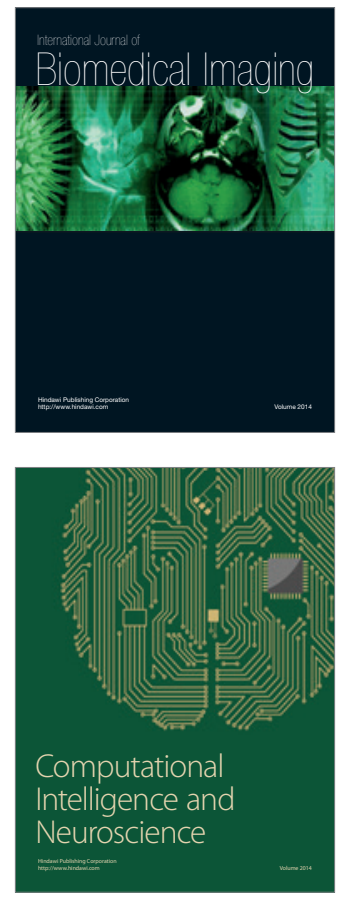
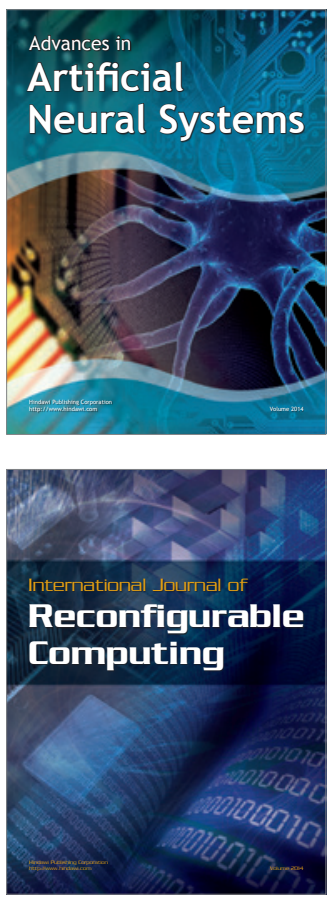
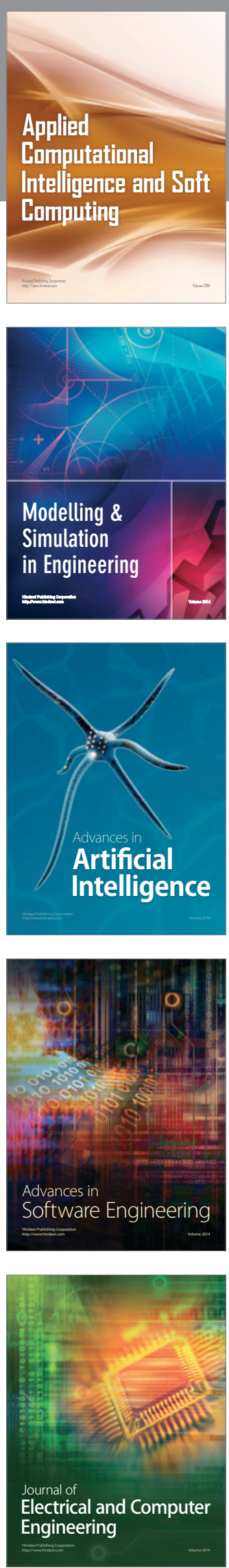